Mathematical Medicine and Biology (2005) 22, 113-128

doi:10.1093/imammb/dqi001

Advance Access publication on March 18, 2005

\title{
Non-linear incidence and stability of infectious disease models
}

\author{
ANDREI KoROBEINIKOV† AND PHILIP K. MAINI \\ Centre for Mathematical Biology, Mathematical Institute, University of Oxford, \\ 24-29 St Giles', Oxford OX1 3LB, UK
}

[Received on 23 February 2004; revised on 28 September 2004]

\begin{abstract}
In this paper we consider the impact of the form of the non-linearity of the infectious disease incidence rate on the dynamics of epidemiological models. We consider a very general form of the non-linear incidence rate (in fact, we assumed that the incidence rate is given by an arbitrary function $f(S, I, N)$ constrained by a few biologically feasible conditions) and a variety of epidemiological models. We show that under the constant population size assumption, these models exhibit asymptotically stable steady states. Precisely, we demonstrate that the concavity of the incidence rate with respect to the number of infective individuals is a sufficient condition for stability. If the incidence rate is concave in the number of the infectives, the models we consider have either a unique and stable endemic equilibrium state or no endemic equilibrium state at all; in the latter case the infection-free equilibrium state is stable. For the incidence rate of the form $g(I) h(S)$, we prove global stability, constructing a Lyapunov function and using the direct Lyapunov method. It is remarkable that the system dynamics is independent of how the incidence rate depends on the number of susceptible individuals. We demonstrate this result using a SIRS model and a SEIRS model as case studies. For other compartment epidemic models, the analysis is quite similar, and the same conclusion, namely stability of the equilibrium states, holds.
\end{abstract}

Keywords: non-linear incidence rate; direct Lyapunov method; endemic equilibrium state; global stability.

\section{Introduction}

Classical epidemiological models postulate that a population is divided into a few classes of epidemiological significance and homogeneously mixed. The spread of an infection is traditionally postulated to occur according to the principle of mass action and associated with it an incidence rate bilinear with respect to the numbers of susceptible and infective individuals (Anderson \& May, 1991; Hethcote, 2000). More specifically, if we denote by $S(t)$ and $I(t)$ the numbers of susceptible and infective individ uals, respectively, and if $\beta$ is the per capita contact rate, then there are $\beta I$ infective contacts. A fraction $S / N$ of these contacts is with susceptible individuals, and hence the total number of new infections is $\beta I S / N$.

However, there is a variety of reasons why this standard bilinear incidence rate may require modification, e.g. the underlying assumption of homogeneous mixing may be invalid. In this case the necessary population structure and heterogeneous mixing may be incorporated into a model with a specific form of non-linear transmission. A non-linear incidence rate can also arise from saturation effects: if the proportion of the infectives in a population is very high, so that exposure to the disease agent is virtually certain, then the transmission rate may respond more slowly than linear to the increase in the number of infectives. This effect was observed, e.g. by Capasso \& Serio (1978) who studied the cholera epidemic spread in Bari in 1973, and by Brown \& Hasibuan (1995) who studied infection of the two-spotted spider

\footnotetext{
†Email: korobein@maths.ox.ac.uk
} 
mites, Tetranychus urticae, with the entomopathogenic fungus, Neozygites floridana. For a discrete-time model, a non-linear incidence rate can also be applied to avoid the possibility of multiple infection of a host.

In recent years, a number of authors have studied epidemiological models with non-linear incidence rates. The most common non-linear incidence rate takes the form $\beta I^{p} S^{q}$ (where $I$ and $S$ are, respectively, the number of infective and susceptible individuals, and $\beta, p$ and $q$ are positive constants). Epidemiological models with this incidence rate have been studied by Liu et al. $(1986,1987)$ and later by Hethcote et al. (1989), Hethcote \& van den Driessche (1991), Derrick \& van den Driessche (1993, 2003), Li \& Muldowney (1995) and many others. More sophisticated forms of the non-linear incidence rate were also considered. For instance, Liu et al. (1986) proposed an incidence rate of the form $k I^{p} S /\left(1+\alpha I^{l}\right)$; Briggs \& Godfray (1995), who studied infection of insects, considered a non-linear pathogen transmission of the form $k S \ln (1+v P / k)$ (where $P$ is the density of the pathogen particles).

Transmission of an infection is unknown in detail and it may be different for different infections. Hence it appears to be reasonable to consider such properties of the system which are robust irrespective of the specific form of incidence rate. In this work, we consider the impact of a general form of non-linearity for the incidence rate assuming that the incidence rate is given by an arbitrary function $f(S, I, N)$. We show that, under a few biologically feasible conditions, a non-linear incidence rate of quite a general form leads to a globally stable system. In Section 2, we introduce the non-linear incidence rate and discuss the conditions it must satisfy. In Section 3, we consider local stability of the equilibrium states. We consider SIRS and SEIRS models as case studies. In Section 4, we apply the direct Lyapunov method to consider global stability of SIR and SEIR models with the incidence rate of the form $g(I) h(S)$. Finally, in Section 5, we discuss our results.

\section{Non-linear incidence rate}

Following the classical assumptions (Anderson \& May, 1991; Hethcote, 2000), we divide a population of constant size $N$ into a number of classes of epidemiological significance. For instance, for SIR and $S I R S$ models, these classes are the susceptibles, the infectives and recovered (or removed) with populations denoted, respectively, by $S, I$ and $R$, such that $S+I+R=N$. For SEIS, SEIR and SEIRS models, a class of exposed hosts (the host in a latent state, i.e. those who are already infected but are not yet infectious) of size $E$ is included, and $S+E+I+R=N$ in this case.

We consider a horizontally transmitted infection. ${ }^{1}$ We postulate that the incidence rate depends on the variables $S, I$ and $N$ only and is given by a function $f(S, I, N)$ continuous together with its derivatives. To be biologically feasible, the function $f(S, I, N)$ must satisfy the conditions

$$
f(S, 0, N)=f(0, I, N)=0
$$

and

$$
\frac{\partial f(S, I, N)}{\partial I}>0, \quad \frac{\partial f(S, I, N)}{\partial S}>0,
$$

for all $S, I>0$. We also assume that the function $f(S, I, N)$ is concave with respect to the variable $I$, i.e.

$$
\frac{\partial^{2} f(S, I, N)}{\partial I^{2}} \leqslant 0, \quad \text { for all } S, I>0 .
$$

\footnotetext{
${ }^{1}$ The horizontal transmission is the transmission from an infective host to a susceptible one, in contrast to the vertical transmission from an infective mother to an unborn or newborn child; see Busenberg \& Cooke (1993).
} 
The first two conditions, (2.1) and (2.2), are obvious. The third one, the inequality (2.3), requires further discussion. Most biologically realistic non-linear incidence rates lead to a function concave with respect to the number of infectives $I$. Thus, the condition (2.3) may be a consequence of saturation effects: when the number of infectives is very high so that exposure to the disease agent is virtually certain, the incidence rate will respond more slowly than linearly to the increase in $I$. Also, a function concave with respect to the number of infectives may arise as a consequence of "psychological" effects: for a very large number of infectives, the probability for an infective to pass the infection further may decrease because the population may tend to reduce the number of contacts. For a discrete-time model, a non-linear incidence rate can be applied to prevent the possibility of a multiple infection of a host. However, this also leads to a disease transmission function concave with respect to the number of infectives. The same result, a concave incidence rate or a disease transmission function, can be obtained if a nonlinear transmission function is introduced into a discrete-time model to capture the non-homogeneity of the population structure, e.g. the negative binomial distribution of the infective contacts (Cullen et al., 2003 ) is associated with a distinctively concave transmission function.

There have been some studies for incidence rates which do not satisfy (2.3), e.g. Liu et al. (1987) suggested that "... a rate of increase faster than linear would be observed under various conditions, for example, if multiple exposures to the disease vector were necessary before infection occurred." However, they provided no example of such disease. Anderson \& May (1991) argue, with reference to Liu et al. (1987), that such a situation can arise “... for viruses that live outside the host for only a short time, that significant incidence levels arise only when virus concentration in the environment exceeds a threshold level; when $I$ is small, the threshold is never exceeded, whereas increasing values of I raises the concentration above threshold." Further they also suggested that “... for some vector-borne disease, the vector may have to attack on average $p$ individuals before acquiring a level of infective stages sufficient to render its next attack effective in transmitting the infection."

Convexity of the incidence rate implies that with growth of the infective population $I$, the probability for a single infective individual to pass the infection further increases. Such an effect is usually associated with some form of cooperation or community effect.

In this study, we restrict ourselves to concave non-linear incidence rates.

Finally, since the basic reproduction number $R_{0}$ of a model, i.e. the average number of secondary cases produced by a single infective introduced into an entirely susceptible population, is proportional to the $\operatorname{limit}_{I \rightarrow 0, S \rightarrow N} \frac{\partial f(S, I, N)}{\partial I}$ (van den Driessche \& Watmough, 2002), it is reasonable to assume that

$$
0<\frac{\partial f(S, I, N)}{\partial I}<\infty
$$

This condition is not needed for our analysis, but we believe that any biologically realistic incidence rate should satisfy this condition. For instance, the incidence rate of the form $\beta I^{p} S^{q}$ does not satisfy this condition for all $p$ unequal to one, leading to models with $R_{0}$ either infinitely large or equal to 0 .

In this paper we show that autonomous compartmental epidemiological models with a non-linear incidence rate satisfying conditions (2.1)-(2.3) and under the constant population size assumption are stable. Such systems have either a unique and stable endemic equilibrium state or no endemic equilibrium state at all; in the latter case, the infection-free equilibrium is stable. In fact, the condition (2.3) is a sufficient condition for the system to be stable. We demonstrate this result using a SIRS model and a SEIRS model as case studies. For other compartment epidemic models, the analysis is quite analogous, and the same conclusion, namely stability of the steady states, holds. 


\section{Stability of SIRS and SEIRS models}

\subsection{SIRS model}

With the incidence rate given by the function $f(S, I, N)$, the standard continuous-time SIRS model (Anderson \& May, 1991; Hethcote, 2000) takes the form

$$
\begin{aligned}
\dot{S} & =\mu N-f(S, I, N)-\mu S+\alpha R, \\
\dot{I} & =f(S, I, N)-\delta I-\mu I, \\
\dot{R} & =\delta I-\alpha R-\mu R .
\end{aligned}
$$

Here $\mu$ is the birth and death rate, $\delta$ is the recovery rate and $\alpha$ is the loss of immunity rate. Following the classical assumptions, we postulate $N=S+I+R$. Hence the third equation may be omitted, and the first two take the form

$$
\begin{aligned}
& \dot{S}=(\alpha+\mu) N-f(S, I, N)-\alpha I-(\alpha+\mu) S, \\
& \dot{I}=f(S, I, N)-(\delta+\mu) I .
\end{aligned}
$$

It is easy to see that when $\alpha=0$ the system (3.1) is a SIR model; when $\alpha \rightarrow \infty$ the system (3.1) reduces to a SIS model. The positive quadrant $\mathbf{R}_{+}^{2}$ of the $S I$ plane is the phase-space of the system.

At equilibrium states of the system, the equalities

$$
\begin{gathered}
f(S, I, N)=(\delta+\mu) I, \\
(\alpha+\mu+\delta) I+(\alpha+\mu) S=(\alpha+\mu) N
\end{gathered}
$$

hold. It is easy to see that the system has an infection-free equilibrium state $Q_{0}=\left(S_{0}, I_{0}\right)$, where $S_{0}=$ $N$ and $I_{0}=0$. Apart from the infection-free equilibrium $Q_{0}$, the system can have positive endemic equilibria states. If $S^{*}$ and $I^{*}$ are coordinates of an endemic equilibrium state $Q^{*}$, then the following Lemma holds.

LEMma 3.1 If the conditions (2.1), (2.2) and (2.3) hold, then at the endemic equilibrium state $Q^{*}=$ $\left(S^{*}, I^{*}\right)$

$$
\frac{\partial f\left(S^{*}, I^{*}, N\right)}{\partial I} \leqslant \delta+\mu,
$$

where the strict equality holds only if

$$
\frac{\partial^{2} f\left(S^{*}, I, N\right)}{\partial I^{2}}=0, \quad \text { for all } I \in\left(0, I^{*}\right)
$$

Proof. We observe that, by (3.1), at the endemic equilibrium state

$$
f\left(S^{*}, I^{*}, N\right)=(\delta+\mu) I^{*}
$$

and twice apply the mean value theorem to the function $\tilde{f}(I)=f\left(S^{*}, I, N\right)$.

Assume that

$$
\frac{\partial f\left(S^{*}, I^{*}, N\right)}{\partial I}=\frac{\mathrm{d} \tilde{f}\left(I^{*}\right)}{\mathrm{d} I}>\delta+\mu .
$$


Then, by the mean value theorem, there is a point $I_{1} \in\left(0, I^{*}\right)$ such that

$$
\frac{\mathrm{d} \tilde{f}\left(I_{1}\right)}{\mathrm{d} I}=\frac{\tilde{f}\left(I^{*}\right)-\tilde{f}(0)}{I^{*}-0}=\delta+\mu .
$$

Furthermore, if (3.4) holds, then there exists a point $I_{0} \in\left(I_{1}, I^{*}\right)$ such that

$$
\frac{\partial^{2} f\left(S^{*}, I_{0}, N\right)}{\partial I^{2}}=\frac{\mathrm{d}^{2} \tilde{f}\left(I_{0}\right)}{\mathrm{d} I^{2}}=\frac{\frac{\mathrm{d} \tilde{f}\left(I^{*}\right)}{\mathrm{d} I}-\frac{\mathrm{d} \tilde{f}\left(I_{1}\right)}{\mathrm{d} I}}{I^{*}-I_{1}}>0 .
$$

This contradicts the hypothesis of the Lemma, hence

$$
\frac{\partial f\left(S^{*}, I^{*}, N\right)}{\partial I} \leqslant \delta+\mu .
$$

Furthermore, the strict equality

$$
\frac{\partial f\left(S^{*}, I^{*}, N\right)}{\partial I}=\delta+\mu
$$

holds only if

$$
\frac{\partial^{2} f(S, I, N)}{\partial I^{2}}=0, \quad \text { for all } I \in\left(0, I^{*}\right), \quad S=S^{*} .
$$

REMARK 3.2 Note that although we fixed the value $S=S^{*}$, we never use this fact in the proof. In fact, the Lemma is valid for all $S, I$ satisfying the equality (3.1). We shall use this fact later.

We define the basic reproduction number, i.e. the expected number of secondary cases produced by one infective host in an entirely susceptible population (van den Driessche \& Watmough, 2002) as

$$
R_{0}=\frac{1}{\delta+\mu} \frac{\partial f\left(S_{0}, I_{0}, N\right)}{\partial I}
$$

LEMMA 3.3 If the conditions (2.1)-(2.3) hold, and if $R_{0}>1$, then, in addition to the infection-free equilibrium state, the system (3.1) has a unique positive endemic equilibrium state $Q^{*}$. If $R_{0} \leqslant 1$, then the infection-free equilibrium $Q_{0}$ is the only non-negative equilibrium of the system.

Proof. Let us consider the curve defined by the equation (3.1) on the SI plane. Since, by (2.2), $\frac{\partial f(S, I, N)}{\partial S}>0$, the implicit function theorem ensures that for all $S$ and $I$ satisfying (3.1), there is a one-to-one function $S=h_{1}(I)$ such that

$$
h_{1}^{\prime}=\frac{\mathrm{d} S}{\mathrm{~d} I}=\frac{\delta+\mu-\frac{\partial f(S, I)}{\partial I}}{\frac{\partial f(S, I)}{\partial S}} .
$$

Lemma 3.1 is valid for all $S, I>0$ such that the equality (3.1) holds, and hence

$$
h_{1}^{\prime}(I)=\frac{\mathrm{d} S}{\mathrm{~d} I} \geqslant 0, \quad \text { for all } I \geqslant 0 .
$$

Equality (3.3) defines a negatively sloped straight line $h_{2}(I)$ on the $S I$ plane (see Fig. 1). Therefore, if $S_{*}=h_{1}(0) \leqslant S_{0}=N$, then there is a unique point of intersection of the curve (3.1) and the straight 


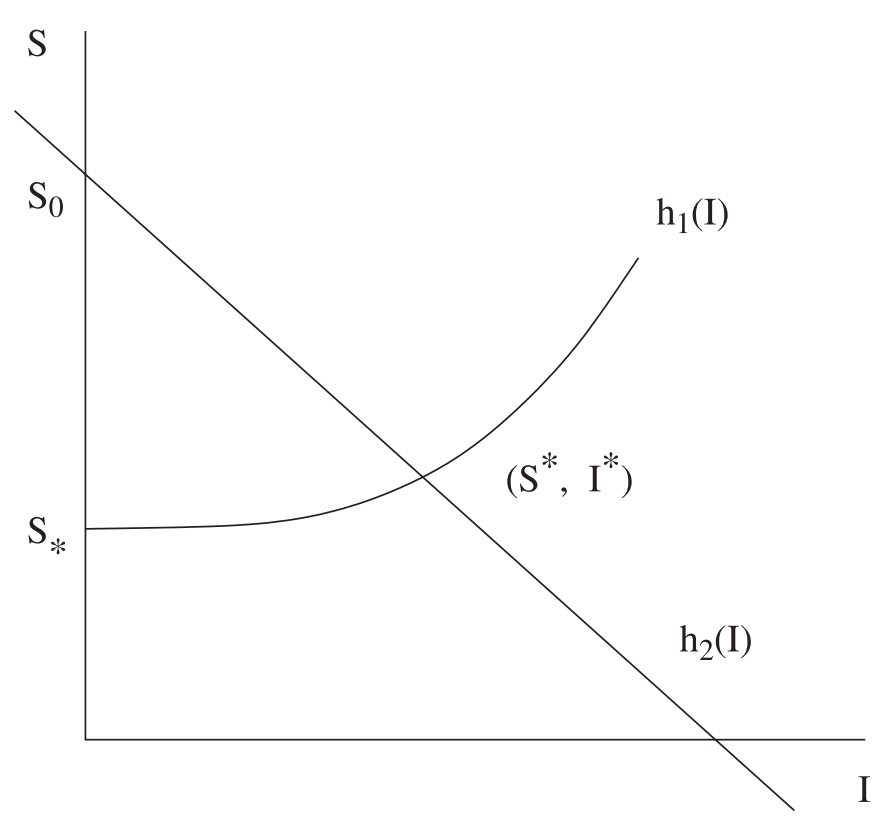

FIG. 1. The functions $S=h_{1}(I)$ and $S=h_{2}(I)$.

line (3.3); if $S_{*}>S_{0}$ the curves (3.1) and (3.3) do not intersect, and there is no positive endemic equilibrium state. Here $S_{*}=h_{1}(0)$ is either a minimal value of $S$ such that

$$
\frac{\partial f(S, 0)}{\partial I}=\delta+\mu
$$

holds, or, if such a value does not exist (as is e.g. in the case of a power function), $S_{*} \equiv 0$. By (2.1) and (2.2), $\frac{\partial f(S, 0)}{\partial I}$ is a non-decreasing function of $S$. Hence the condition

$$
\frac{\partial f(N, 0)}{\partial I}>\delta+\mu
$$

is sufficient to ensure that $S_{*}<S_{0}=N$.

The following theorem is a straightforward consequence of Lemma 3.1.

\section{THEOREM 3.4}

(i) If the incidence rate $f(S, I, N)$ satisfies the conditions (2.1), (2.2) and (2.3), and if $R_{0}>1$, then the endemic equilibrium state $Q^{*}=\left(S^{*}, I^{*}\right)$ of the system (3.1) is asymptotically stable.

(ii) If $R_{0} \leqslant 1$, then there is no endemic equilibrium state, and the infection-free equilibrium state is asymptotically stable.

Proof.

(i) For the Jacobian $J$ of the system (3.1), the conditions (2.2) and Lemma 3.1 ensure that at the fixed point $Q^{*}=\left(S^{*}, I^{*}\right)$,

$$
\operatorname{det} J=(\alpha+\mu)\left(\delta+\mu-\frac{\partial f}{\partial I}\right)+(\alpha+\delta+\mu) \frac{\partial f}{\partial S} \geqslant(\alpha+\delta+\mu) \frac{\partial f}{\partial S}>0
$$


and

$$
\lambda_{1}+\lambda_{2}=\operatorname{tr} J=\frac{\partial f}{\partial I}-\delta-\frac{\partial f}{\partial S}-\alpha-2 \mu \leqslant-\left(\frac{\partial f}{\partial S}+\alpha+\mu\right)<0 .
$$

Hence the real parts of the eigenvalues are negative, and the fixed point $Q^{*}$ is asymptotically stable.

(ii) At the infection-free equilibrium $Q_{0}$, by (2.1) and (3.5), $\frac{\partial f\left(S_{0}, I_{0}\right)}{\partial S}=0$ and $\frac{\partial f\left(S_{0}, I_{0}\right)}{\partial I}=(\delta+\mu) R_{0}$. Hence at this point the eigenvalues are

$$
\lambda_{1}=-\alpha-\mu<0, \quad \lambda_{2}=\left(R_{0}-1\right)(\delta+\mu),
$$

i.e. for $R_{0}<1$ the fixed point $Q_{0}$ is a stable node, at $R_{0}=1$ a transcritical bifurcation occurs and when $R_{0}>1$ the fixed point is a saddle point.

The following corollaries are obvious.

COROLlary 3.5 For instability of the endemic equilibrium state, the incidence rate $f(S, I, N)$ must be necessarily convex with respect to $I$ at least in some points of the interval $\left(0, I^{*}\right)$.

Proof. By the proof of Theorem 3.4,

$$
\lambda_{1} \cdot \lambda_{2}=\operatorname{det} J=(\alpha+\mu)\left(\delta+\mu-\frac{\partial f}{\partial I}\right)+(\alpha+\delta+\mu) \frac{\partial f}{\partial S}
$$

and

$$
\lambda_{1}+\lambda_{2}=\operatorname{tr} J=\frac{\partial f}{\partial I}-\delta-\mu-\frac{\partial f}{\partial S}-\alpha-\mu .
$$

It is obvious that

$$
\frac{\partial f\left(S^{*}, I^{*}\right)}{\partial I}-\delta-\mu>0
$$

must necessarily hold to have positive real parts of one or both eigenvalues.

COROLlary 3.6 The system dynamics is independent of how the incidence rate $f(S, I, N)$ depends on the variable $S$.

Proof. If the incidence rate $f(S, I, N)$ satisfies the conditions (2.1), (2.2) and (2.3), then it is obvious that the signs of real parts of the eigenvalues do not depend on the value of $\frac{\partial f}{\partial S}$.

If the incidence rate is convex, then the positive equilibrium state may not be unique. The dynamics of the system in this case can be very complex.

\subsection{SEIRS model}

Addition of the exposed state and associated with it the exposed compartment $E$ does not affect the system stability. Let us consider a more complicated model, namely a SEIRS model. If the incidence rate is given by a function $f(S, I, N)$, then the differential equations of the model are

$$
\begin{aligned}
\dot{S} & =(\alpha+\mu) N-f(S, I, N)-\alpha I-\alpha E-(\alpha+\mu) S, \\
\dot{E} & =f(S, I, N)-\theta E-\mu E, \\
\dot{I} & =\theta E-\delta I-\mu I .
\end{aligned}
$$


Here the fourth equation, the equation for the recovered population $R$, is omitted-the constant population size assumption allows us to do this. We assume here that the function $f(S, I, N)$ satisfies the conditions (2.1), (2.2) and (2.3), and does not depend on the exposed population $E$. We define the basic reproduction number of the system as

$$
R_{0}=\frac{\theta}{(\theta+\mu)(\delta+\mu)} \frac{\partial f\left(S_{0}, I_{0}, N\right)}{\partial I} .
$$

Equilibrium states of the system satisfy

$$
\begin{aligned}
f(S, I, N) & =\frac{\theta+\mu}{\theta}(\delta+\mu) I, \\
N & =S+\left(1+\frac{\delta}{\alpha+\mu}+\frac{\delta+\mu}{\theta}\right) I, \\
(\delta+\mu) I & =\theta E .
\end{aligned}
$$

Since, by (2.1), $f(S, 0, N) \equiv 0$, the infection-free equilibrium state $Q_{0}$ has the coordinates $S_{0}=N$, $E_{0}=I_{0}=0$. The system also can have endemic equilibrium states.

It is easy to see that with minor changes, Lemmas 3.1 and 3.3 are valid for the system (3.6) as well.

LEMmA 3.7 If the conditions (2.1), (2.2) and (2.3) hold, and if $Q^{*}=\left(S^{*}, E^{*}, I^{*}\right)$ is the endemic equilibrium state, then

$$
\frac{\partial f\left(S^{*}, I^{*}, N\right)}{\partial I} \leqslant \frac{\theta+\mu}{\theta}(\delta+\mu) .
$$

The strict equality holds only if

$$
\frac{\partial^{2} f(S, I, N)}{\partial I^{2}}=0, \quad \text { for all } I \in\left(0, I^{*}\right), S=S^{*} .
$$

LEMMA 3.8 If the incidence rate $f(S, I, N)$ satisfies the conditions (2.1), (2.2) and (2.3), and if $R_{0}>1$, then the system (3.6) has the unique infection-free equilibrium state $Q_{0}=\left(S_{0}, E_{0}, I_{0}\right)$ and the unique positive endemic equilibrium state $Q^{*}=\left(S^{*}, E^{*}, I^{*}\right)$. If $R_{0} \leqslant 1$, then the system (3.6) has no endemic equilibrium state. In this case the only non-negative equilibrium of the system is the infection-free equilibrium $Q_{0}$.

We omit the proofs of these Lemmas since these are the same as for Lemmas 3.1 and 3.3. The following Theorem is a consequence of Lemma 3.7.

\section{THEOREM 3.9}

(i) If the incidence rate $f(S, I, N)$ satisfies the conditions (2.1), (2.2) and (2.3), and $R_{0}>1$, then the endemic equilibrium state $Q^{*}$ of the system (3.6) is asymptotically stable.

(ii) If $R_{0} \leqslant 1$, then there is no endemic equilibrium state, and the infection-free equilibrium is asymptotically stable.

Proof.

(i) The Jacobian of the system (3.6) is

$$
J=\left[\begin{array}{ccc}
-\left(\frac{\partial f}{\partial S}+\alpha+\mu\right) & -\alpha & -\left(\frac{\partial f}{\partial I}+\alpha\right) \\
\frac{\partial f}{\partial S} & -(\theta+\mu) & \frac{\partial f}{\partial I} \\
0 & \theta & -(\delta+\mu)
\end{array}\right]
$$


By the Routh-Hurwitz Criterion (Gantmacher, 1959), the eigenvalues of the matrix have negative real parts if and only if the inequalities

$$
a_{1}, a_{2}, a_{3}>0, \quad \text { and } \quad \Delta_{2} \equiv a_{1} a_{2}-a_{3}>0
$$

hold for the coefficients of the characteristic equation

$$
\lambda^{3}+a_{1} \lambda^{2}+a_{2} \lambda+a_{3}=0 .
$$

Lemma 3.7 and condition (2.2) ensure that all these inequalities hold at $Q^{*}$. Indeed, by Lemma 3.7,

$$
\frac{\partial f\left(S^{*}, I^{*}, N\right)}{\partial I} \leqslant \frac{\theta+\mu}{\theta}(\delta+\mu)
$$

and hence

$$
\begin{aligned}
a_{1} & =\frac{\partial f}{\partial S}+\alpha+\delta+\theta+3 \mu>0, \\
a_{2} & =(\alpha+\theta+\delta+2 \mu) \frac{\partial f}{\partial S}+(\alpha+\mu)(\delta+\theta+2 \mu)+\left((\delta+\mu)(\theta+\mu)-\theta \frac{\partial f}{\partial I}\right) \\
& >(\alpha+\theta+\delta+2 \mu) \frac{\partial f}{\partial S}+(\alpha+\mu)(\delta+\theta+2 \mu)>0
\end{aligned}
$$

and

$$
\begin{aligned}
a_{3} & =[(\delta+\mu)(\theta+\mu)+\alpha(\delta+\theta+\mu)] \frac{\partial f}{\partial S}+(\alpha+\mu)\left((\delta+\mu)(\theta+\mu)-\theta \frac{\partial f}{\partial I}\right) \\
& >[(\delta+\mu)(\theta+\mu)+\alpha(\delta+\theta+\mu)] \frac{\partial f}{\partial S}>0 .
\end{aligned}
$$

For $\Delta_{2}$ we obtain

$$
\begin{aligned}
\Delta_{2}= & a_{1} a_{2}-a_{3} \\
= & a_{2}\left(\frac{\partial f}{\partial S}+\delta+\theta+2 \mu\right)+(\alpha+\mu)^{2} \frac{\partial f}{\partial S}+(\alpha+\mu)^{2}(\delta+\theta+2 \mu) \\
& -(\delta+\mu)(\theta+\mu) \frac{\partial f}{\partial S} \\
\geqslant & (\alpha+\mu)(\delta+\theta+2 \mu) a_{1}+(\alpha+\theta+\delta+2 \mu)\left(\frac{\partial f}{\partial S}\right)^{2} \\
& +\left((\theta+\mu)(\alpha+\theta+\delta+2 \mu)+(\alpha+\mu)^{2}+(\delta+\mu)(\alpha+\delta+\mu)\right) \frac{\partial f}{\partial S}>0 .
\end{aligned}
$$

Therefore, all three roots of the characteristic equation have negative real parts, and hence the endemic equilibrium state $Q^{*}$ is asymptotically stable.

(ii) At the infection-free equilibrium $Q_{0}$,

$$
\begin{aligned}
& a_{1}=\alpha+\delta+\theta+3 \mu, \\
& a_{2}=(\alpha+\mu)(\delta+\theta+2 \mu)+(\delta+\mu)(\theta+\mu)\left(1-R_{0}\right), \\
& a_{3}=(\alpha+\mu)(\delta+\mu)(\theta+\mu)\left(1-R_{0}\right)
\end{aligned}
$$


and

$$
\Delta_{2}=a_{1} a_{2}-a_{3}=(\delta+\theta+2 \mu) a_{2}+(\alpha+\mu)^{2}(\delta+\theta+2 \mu),
$$

i.e. $R_{0}<1$ ensures that the infection-free equilibrium is asymptotically stable. At $R_{0}=1 \mathrm{a}$ transcritical bifurcation occurs, the equilibrium $Q_{0}$ loses stability and it is unstable for all $R_{0}>1$. This completes the proof.

\section{Global properties of SIR and SEIR systems}

The majority of the incidence rates in the literature can be represented as a product of two functions, $f(S, I)=h(S) g(I)$, where $h$ depends only on $S$, and $g$ depends only on $I$. It is easy to see that all the incidence rates mentioned in Section 1, including the mass-action incidence and the power incidence, can be represented in this form. For the incidence rate of the form $h(S) g(I)$ satisfying the conditions (2.1)-(2.3), the direct Lyapunov method enables us to prove global stability for some models. The direct Lyapunov method so far is the most effective method of global analysis. However, the method requires an auxiliary function with specific properties, the so-called Lyapunov function. Here we construct Lyapunov functions for SIR and SEIR systems with the incidence rate of the form $h(S) g(I)$.

For simplicity, we normalize the population size $N$ to 1 ; i.e. now $S, E, I$ and $R$ are, respectively, the fractions of the susceptibles, the exposed, the infectives and the recovered in the population, and $S+E+I+R=1$ holds. Then the basic $S I R$ model is

$$
\begin{aligned}
& \dot{S}=\mu-h(S) g(I)-\mu S, \\
& \dot{I}=h(S) g(I)-(\delta+\mu) I,
\end{aligned}
$$

and the basic SEIR model is

$$
\begin{aligned}
& \dot{S}=\mu-h(S) g(I)-\mu S, \\
& \dot{E}=h(S) g(I)-(\theta+\mu) E, \\
& \dot{I}=\theta E-(\delta+\mu) I .
\end{aligned}
$$

Here we omit the equations for the recovered population $R$, and as usual we assume that the incidence rate satisfies the conditions (2.1), (2.2) and (2.3). The condition (2.3) ensures that each of these systems has two equilibrium states: an infection-free equilibrium $Q_{0}$ with the coordinates $S_{0}=1, E_{0}=I_{0}=0$, and an endemic equilibrium state $Q^{*}=\left(S^{*}, E^{*}, I^{*}\right)$, such that

$$
B(\delta+\mu) I^{*}=h\left(S^{*}\right) g\left(I^{*}\right), \quad \mu S^{*}+B(\delta+\mu) I^{*}=\mu, \quad B(\delta+\mu) I^{*}=(\theta+\mu) E^{*} .
$$

Here $B=(\theta+\mu) / \theta$ for the SEIR model, and $B=1$ for the SIR model.

For these SIR and SEIR models, we construct a Lyapunov function of the form

$$
V(S, E, I)=S-h\left(S^{*}\right) \int_{a}^{S} \frac{\mathrm{d} \tau}{h(\tau)}+B\left(I-g\left(I^{*}\right) \int_{a}^{I} \frac{\mathrm{d} \tau}{g(\tau)}\right)+C\left(E-E^{*} \ln E\right) .
$$

Here $C=1$ for the SEIR model and $C=0$ for the SIR model, and $B$ is defined above. The parameter $a$, such that $0<a \ll 1$, is an arbitrary positive constant which is not fixed and can be made sufficiently small. The function $V(S, E, I)$ is defined and continuous for all $S, E, I \geqslant a$. 
The endemic equilibrium state $Q^{*}$ is the only extremum and global minimum of the function. Indeed, the function $V(S, E, I)$ satisfies

$$
\frac{\partial V}{\partial S}=1-\frac{h\left(S^{*}\right)}{h(S)}, \quad \frac{\partial V}{\partial E}=C\left(1-\frac{E^{*}}{E}\right), \quad \frac{\partial V}{\partial I}=B\left(1-\frac{g\left(I^{*}\right)}{g(I)}\right)
$$

and it is easy to see that $Q^{*}$ is a stationary point of the function. Since the functions $h(S)$ and $g(I)$ grow monotonically, the partial derivatives $\frac{\partial V}{\partial S}$ and $\frac{\partial V}{\partial I}$ grow monotonically as well, and hence $Q^{*}$ is the only extremum of the function. Furthermore, since

$$
\frac{\partial^{2} V}{\partial S^{2}}=\frac{h\left(S^{*}\right) h^{\prime}(S)}{(h(S))^{2}}>0, \quad \frac{\partial^{2} V}{\partial E^{2}}=C \frac{E^{*}}{E^{2}}>0 \quad \text { and } \quad \frac{\partial^{2} V}{\partial I^{2}}=B \frac{g\left(I^{*}\right) g^{\prime}(I)}{(g(I))^{2}}>0,
$$

whereas

$$
\frac{\partial^{2} V}{\partial S \partial E}=\frac{\partial^{2} V}{\partial S \partial I}=\frac{\partial^{2} V}{\partial E \partial I}=0
$$

the point $Q^{*}$ is the minimum. The point $Q^{*}$ is the only internal stationary point of the function, it is a minimum, and $V(S, E, I) \rightarrow \infty$ at the boundary. Consequently, $Q^{*}$ is the global minimum, the function is bounded from below. Hence the function $V(S, E, I)$ is indeed a Lyapunov function (Lyapunov, 1992).

In the case of the standard bilinear incidence rate $\beta S I$, the function $V(S, E, I)$ takes the form

$$
V(S, I)=S-S^{*} \ln S+B\left(I-I^{*} \ln I\right)+C\left(E-E^{*} \ln E\right) .
$$

Lyapunov functions of this type were constructed for three- and four-compartmental models with the bilinear incidence rate (Korobeinikov \& Wake, 2002; Korobeinikov, 2004). For the incidence rate of the form $\beta I^{p} S^{q}$, the function $V(S, E, I)$ takes the form

$$
V(S, E, I)=S+\frac{S^{*}}{q-1}\left(\frac{S^{*}}{S}\right)^{q-1}+B\left(I+\frac{I^{*}}{p-1}\left(\frac{I^{*}}{I}\right)^{p-1}\right)+C\left(E-E^{*} \ln E\right)
$$

such a function was constructed for SIR and SEIR models (Korobeinikov \& Maini, 2004). The function (4.4) is a generalization of these Lyapunov functions to the case of the non-linear incidence rate $h(S) g(I)$.

The following Theorem provides global properties of the systems (4.1) and (4.2).

\section{THEOREM 4.1}

(i) If the incidence rate satisfies the conditions (2.1), (2.2) and (2.3), and if $R_{0}>1$, then the endemic equilibrium state $Q^{*}$ is globally asymptotically stable.

(ii) If $R_{0} \leqslant 1$, then there is no positive equilibrium state $Q^{*}$, and the infection-free equilibrium state $Q_{0}$ is globally asymptotically stable.

\section{Proof.}

(i) In the case of the SEIR system (4.2), using the equalities

$$
h\left(S^{*}\right) g\left(I^{*}\right)=B(\delta+\mu) I^{*}, \quad \mu=\mu S^{*}+B(\delta+\mu) I^{*}, \quad(\theta+\mu) E^{*}=B(\delta+\mu) I^{*},
$$


for the equilibrium state $Q^{*}$, the Lyapunov function $V(S, E, I)$ satisfies

$$
\begin{aligned}
\frac{\mathrm{d} V(S, E, I)}{\mathrm{d} t}= & \mu-h(S) g(I)-\mu S-\mu \frac{h\left(S^{*}\right)}{h(S)}+h\left(S^{*}\right) g(I)+\mu S \frac{h\left(S^{*}\right)}{h(S)} \\
& +h(S) g(I)-(\theta+\mu) E-h(S) g(I) \frac{E^{*}}{E}+(\theta+\mu) E^{*} \\
& +B\left(\theta E-(\delta+\mu) I-\theta E \frac{g\left(I^{*}\right)}{g(I)}+(\delta+\mu) I \frac{g\left(I^{*}\right)}{g(I)}\right) \\
= & \mu S^{*}\left(1-\frac{S}{S^{*}}-\frac{h\left(S^{*}\right)}{h(S)}+\frac{S}{S^{*}} \frac{h\left(S^{*}\right)}{h(S)}\right) \\
& -B(\delta+\mu) I^{*}\left(\frac{h\left(S^{*}\right)}{h(S)}+\frac{h(S) g(I) E^{*}}{h\left(S^{*}\right) g\left(I^{*}\right) E}+\frac{g\left(I^{*}\right) E}{g(I) E^{*}}-2\right) \\
& +B(\delta+\mu) I^{*}\left(\frac{g(I)}{g\left(I^{*}\right)}-\frac{I}{I^{*}}+\frac{I}{I^{*}} \frac{g\left(I^{*}\right)}{g(I)}\right) \\
= & \mu S^{*}\left(1-\frac{S}{S^{*}}\right)\left(1-\frac{h\left(S^{*}\right)}{h(S)}\right) \\
& -B(\delta+\mu) I^{*}\left(\frac{h\left(S^{*}\right)}{h(S)}+\frac{h(S) g(I) E^{*}}{h\left(S^{*}\right) g\left(I^{*}\right) E}+\frac{g\left(I^{*}\right) E}{g(I) E^{*}}-3\right) \\
& +B(\delta+\mu) I^{*}\left(1-\frac{g\left(I^{*}\right)}{g(I)}\right)\left(\frac{g(I)}{g\left(I^{*}\right)}-\frac{I}{I^{*}}\right) .
\end{aligned}
$$

The concavity of the function $g(I)$ ensures that $\frac{\mathrm{d} V}{\mathrm{~d} t} \leqslant 0$, for all $S, E, I>a$, where the equality holds only at the point $Q^{*}$. Indeed, since the arithmetical mean is greater than or equal to the geometrical mean,

$$
\frac{h\left(S^{*}\right)}{h(S)}+\frac{h(S) g(I) E^{*}}{h\left(S^{*}\right) g\left(I^{*}\right) E}+\frac{g\left(I^{*}\right) E}{g(I) E^{*}} \geqslant 3, \quad \text { for all } S, E, I>0 .
$$

Furthermore,

$$
\left(1-\frac{S}{S^{*}}\right)\left(1-\frac{h\left(S^{*}\right)}{h(S)}\right) \leqslant 0, \quad \text { for all } S>0,
$$

since for a monotonically growing function $h(S), h(S) \geqslant h\left(S^{*}\right)$ when $S \geqslant S^{*}$ and $h(S) \leqslant h\left(S^{*}\right)$ when $S \leqslant S^{*}$. Also

$$
\left(1-\frac{g\left(I^{*}\right)}{g(I)}\right)\left(\frac{g(I)}{g\left(I^{*}\right)}-\frac{I}{I^{*}}\right) \leqslant 0,
$$

if

$$
\begin{cases}g(I) / g\left(I^{*}\right) \geqslant I / I^{*}, & \text { for } 0<I \leqslant I^{*} \\ g(I) / g\left(I^{*}\right) \leqslant I / I^{*}, & \text { for } I \geqslant I^{*}\end{cases}
$$

It is easy to see that this condition holds for all concave functions $g(I)$ (Fig. 2). Since the parameter $a$ can be chosen sufficiently small, by the asymptotic stability theorem, the SEIR system (4.2) is globally asymptotically stable in $\mathbf{R}_{+}^{3}$ (Barbashin, 1970; La Salle \& Lefschetz, 1961). 


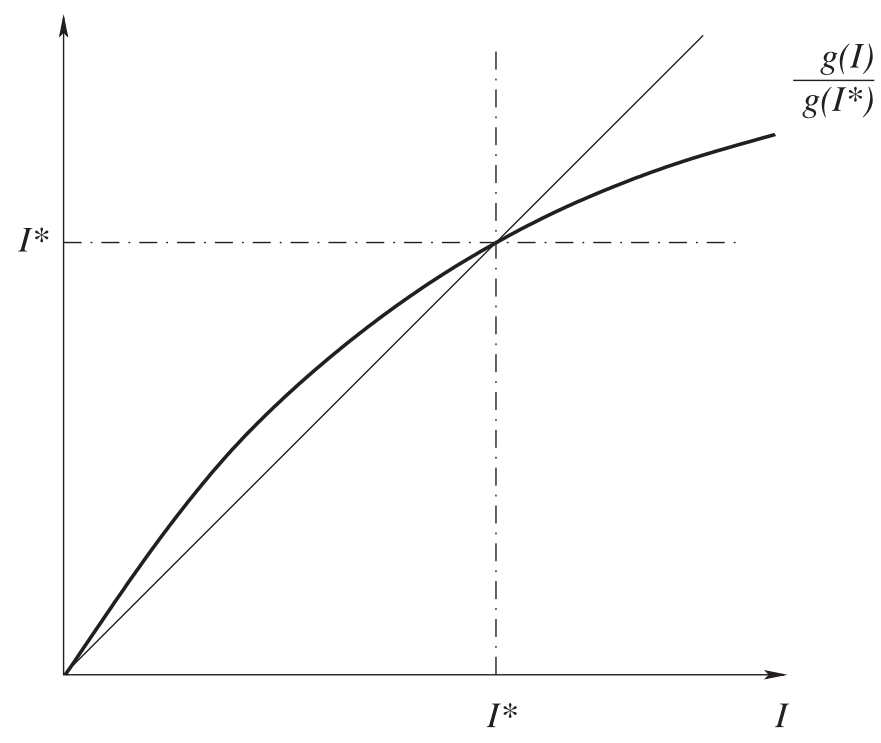

FIG. 2. A concave function $g(I)$.

In the case of the SIR system (4.1), the Lyapunov function (4.4) satisfies

$$
\begin{aligned}
\frac{\mathrm{d} V(S, I)}{\mathrm{d} t}= & -(\delta+\mu) I^{*} \frac{h\left(S^{*}\right)}{h(S)}\left(1-\frac{h(S)}{h\left(S^{*}\right)}\right)^{2}+\mu S^{*}\left(1-\frac{S}{S^{*}}\right)\left(1-\frac{h\left(S^{*}\right)}{h(S)}\right) \\
& -(\delta+\mu) I^{*}\left(\frac{g\left(I^{*}\right)}{g(I)}-1\right)\left(\frac{g(I)}{g\left(I^{*}\right)}-\frac{I}{I^{*}}\right),
\end{aligned}
$$

i.e. in this case $\frac{\mathrm{d} V}{\mathrm{~d} t} \leqslant 0$, for all $S, I>a$ as well, and hence the endemic equilibrium state is globally asymptotically stable.

(ii) To prove global stability of the infection-free equilibrium states $Q_{0}$, we consider the Lyapunov function of the form

$$
U(S, I)=S-h\left(S_{0}\right) \int_{a}^{S} \frac{\mathrm{d} \tau}{h(\tau)}+C E+B I
$$

(where as above $C=1$ and $B=(\theta+\mu) / \theta$ for the SEIR model and $C=0$ and $B=1$ for the SIR model). In the case of the SEIR system (4.2), the Lyapunov function satisfies

$$
\begin{aligned}
\frac{\mathrm{d} U(S, E, I)}{\mathrm{d} t}= & \mu-h(S) g(I)-\mu S-\mu \frac{h\left(S_{0}\right)}{h(S)}+h\left(S_{0}\right) g(I)+\mu S \frac{h\left(S_{0}\right)}{h(S)} \\
& +h(S) g(I)-(\theta+\mu) E+(\theta+\mu) E-B(\delta+\mu) I \\
= & \mu(1-S)\left(1-\frac{h\left(S_{0}\right)}{h(S)}\right)+B(\delta+\mu) I\left(\frac{h\left(S_{0}\right)}{B(\delta+\mu)} \frac{g(I)}{I}-1\right) .
\end{aligned}
$$




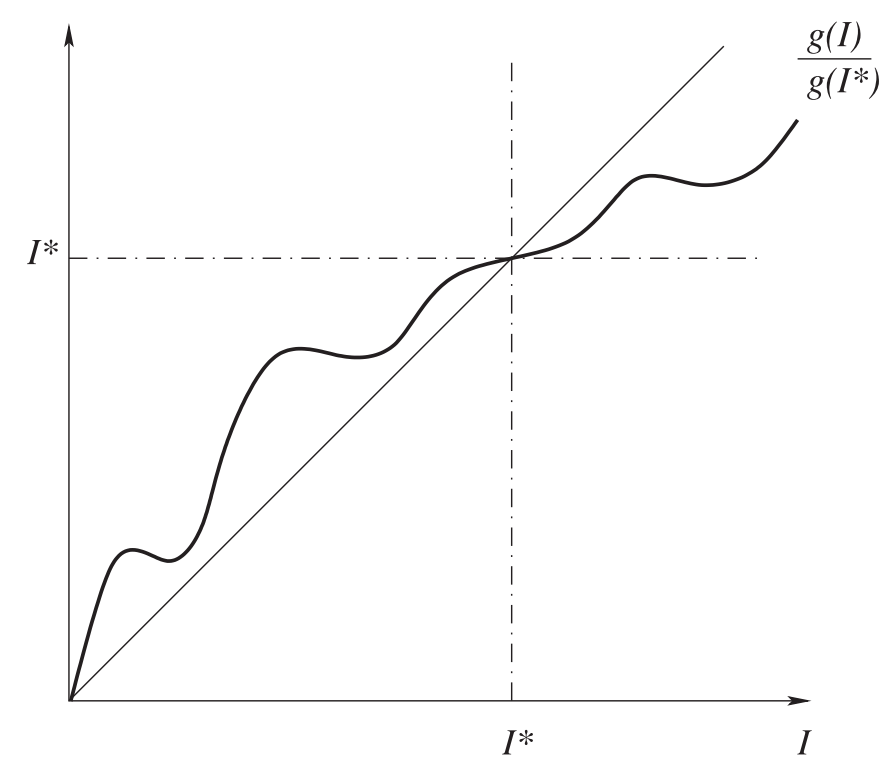

FIG. 3. Non-concave and non-monotonic function $g(I)$ satisfying the condition (4.5).

Here

$$
(1-S)\left(1-\frac{h\left(S_{0}\right)}{h(S)}\right) \leqslant 0, \quad \text { for all } S>0,
$$

and the conditions (2.3) and (2.1) ensure that $\frac{g(I)}{I} \leqslant \frac{\partial g(0)}{\partial I}$, for all $I>0$. Hence using (3.7),

$$
\frac{h\left(S_{0}\right)}{B(\delta+\mu)} \frac{g(I)}{I} \leqslant \frac{h\left(S_{0}\right)}{B(\delta+\mu)} \frac{\partial g(0)}{\partial I}=R_{0} .
$$

Therefore, $R_{0} \leqslant 1$ ensures that $\frac{\mathrm{d} V}{\mathrm{~d} t} \leqslant 0$, for all $S, E, I>a$, and hence by the asymptotic stability theorem, the equilibrium state $Q_{0}$ is globally asymptotically stable in this case.

The proof for the SIR system is the same, and we omit it.

REMARK 4.2 The concavity of the function $g(I)$ is a sufficient but not necessary condition for the global stability of the systems (4.1) and (4.2). It is obvious that a weaker condition (4.5) suffices for the global stability. The biologically feasible condition of monotonicity of the function $g(I)$ is not necessary to satisfy the condition (4.5) either. Figure 3 shows a non-concave and non-monotonic function satisfying the condition (4.5) and providing a unique extremum for $V(S, E, I)$, and hence ensuring global stability of the systems.

We have also to stress that the system dynamics does not depend on the function $h(S)$ at all and is determined by the function $g(I)$ only. The biologically feasible monotonicity of the function $h(S)$ is sufficient for global stability. Furthermore, the monotonicity is not a necessary condition either: in fact, for global stability, it is necessary that $h(S)>h\left(S^{*}\right)$ for all $S>S^{*}$, and $h(S)<h\left(S^{*}\right)$ for all $S<S^{*}$; this also ensures the uniqueness of the extremum of the function $V(S, E, I)$. 


\section{Discussion and conclusion}

In this paper we considered the dynamics of epidemiological models with incidence rate $f(S, I, N)$ concave with respect to the number of infectives, i.e. satisfying the condition

$$
\frac{\partial^{2} f(S, I, N)}{\partial I^{2}} \leqslant 0
$$

The main result of this paper is that this condition, the concavity of the incidence rate, ensures uniqueness of the endemic equilibrium state and its stability. We should stress that this result is valid for autonomous models and with the assumption of constant population size.

It also follows that to have unstable non-trivial (positive) equilibria, the incidence rate $f(S, I, N)$ must necessarily be convex with respect to the variable $I$, at least in some points.

\section{Acknowledgement}

A. K. is supported by the Foundation for Research, Science and Technology of New Zealand, through Project contract UOXX0101.

\section{REFERENCES}

Anderson, R. M. \& MAY, R. M. (1991) Infectious Diseases in Humans: Dynamics and Control. Oxford: Oxford University Press.

BARBASHIN, E. A. (1970) Introduction to the Theory of Stability. Groningen: Wolters-Noordhoff.

BRIgGS, C. J. \& Godfray, H. C. J. (1995) The dynamics of insect-pathogen interactions in stage-structured populations. Am. Nat., 145, 855-887.

BRown, G. C. \& HASIBUAN, R. (1995) Conidial discharge and transmission efficiency of Neozygites floridana, an entomopathogenic fungus infecting two-spotted spider mites under laboratory conditions. J. Invertebr. Pathol., $\mathbf{6 5}, 10-16$.

Busenberg, S. \& CoOKe, K. (1993) Vertically Transmitted Diseases. Berlin: Springer.

CAPAsso, V. \& Serio, G. (1978) A generalisation of the Kermack-McKendrick deterministic epidemic model. Math. Biosci., 42, 43-61.

Cullen, R. M., Korobeinikov, A. \& Walker, W. J. (2003) Seasonality and critical community size for infectious diseases. ANZIAM J., 44, 501-512.

DERRICK, W. R. \& VAN DEN DRIESSCHE, P. (1993) A disease transmission model in a nonconstant population. J. Math. Biol., 31, 495-512.

DERRICK, W. R. \& VAN DEN DRIESSCHE, P. (2003) Homoclinic orbits in a disease transmission model with nonlinear incidence and nonconstant population. Discrete Contin. Dyn. Syst. Ser. B, 3, 299-309.

Gantmacher, F. R. (1959) The Theory of Matrices. New York: Chelsea Publishers.

Hethcote, H. W. (2000) The mathematics of infectious diseases. SIAM Rev., 42, 599-653.

Hethcote, H. W., Lewis, M. A. \& VAn Den Driessche, P. (1989) An epidemiological model with delay and a nonlinear incidence rate. J. Math. Biol., 27, 49-64.

Hethcote, H. W. \& VAN DEN DRIESSche, P. (1991) Some epidemiological models with nonlinear incidence. J. Math. Biol., 29, 271-287.

KorobeInikov, A. (2004) Lyapunov functions and global properties for SEIR and SEIS epidemic models. IMA Math. Med. Biol., 21, 75-83.

Korobeinikov, A. \& MAINI, P. K. (2004) A Lyapunov function and global properties for SIR and SEIR epidemiological models with nonlinear incidence. Math. Biosci. Eng., 1, 57-60.

Korobeinikov, A. \& WAKe, G. C. (2002) Lyapunov functions and global stability for SIR, SIRS and SIS epidemiological models. Appl. Math. Lett., 15, 955-961. 
La SAlle, J. \& Lefschetz, S. (1961) Stability by Liapunov's direct method. New York: Academic Press.

LI, M. Y. \& MULDowneY, J. S. (1995) Global stability for the SEIR model in epidemiology. Math. Biosci., 125, $155-164$.

Liu, W. M., Hethcote, H. W. \& Levin, S. A. (1987) Dynamical behavior of epidemiological models with nonlinear incidence rates. J. Math. Biol., 25, 359-380.

LIU, W. M., LEVIN, S. A. \& ISAWA, Y. (1986) Influence of nonlinear incidence rates upon the behaviour of SIRS epidemiological models. J. Math. Biol., 23, 187-204.

Lyapunov, A. M. (1992) The General Problem of the Stability of Motion. London: Taylor \& Francis.

VAN DEN DRIESSCHE, P. \& WATMOUGH, J. (2002) Reproduction numbers and sub-threshold endemic equilibria for compartmental models of disease transmission. Math. Biosci., 180, 29-48. 